\title{
METABÓLITOS SECUNDÁRIOS DA FAMÍLIA BROMELIACEAE
}

Liliana Maria Manetti

Biotecnologia Aplicada à Agricultura, Instituto de Ciências Exatas, Agrárias, Tecnológicas e Geociências, Universidade Paranaense, 87502-210 Umuarama - PR, Brasil

Rosemeres Horwat Delaporte

Departamento de Farmácia, Instituto de Ciências Biológicas, Médicas e da Saúde, Universidade Paranaense, 87502-210 Umuarama - PR, Brasil

\section{Antonio Laverde Jr.*}

Biotecnologia Aplicada à Agricultura, Instituto de Ciências Exatas, Agrárias, Tecnológicas e Geociências; Departamento de Farmácia, Instituto de Ciências Biológicas, Médicas e da Saúde, Universidade Paranaense, 87502-210 Umuarama - PR, Brasil

Recebido em 23/8/08; aceito em 13/2/09; publicado na web em 4/8/09

\begin{abstract}
SECONDARY METABOLITES FROM BROMELIACEAE FAMILY. This review describes aspects of the Bromeliaceae family dealing the traditional applications, biological activities and distribution of secondary metabolites in distinct subfamilies. Some species are used with medicinal purposed in the treatment of respiratory, diabetes or inflammation diseases, and gastrointestinal disorders. Special emphasis on cycloartane triterpenoids and flavonoids, typical metabolites of this family, are presented. Bromeliaceae is unique amongst the monocotyledons in the frequency and variety of flavonoids with hydroxylation or methoxylation at the 6-position. Other compound classes as steroids, hidroxycinnamic acids, phenylpropane diglycerides, lignans, are presented.
\end{abstract}

Keywords: Bromeliaceae; cycloartane triterpenoides; flavonoids.

\section{INTRODUÇÃO}

A família Bromeliaceae inclui cerca de 56 gêneros e mais de 2700 espécies, divididos em três subfamílias: Pitcairnioideae, Bromelioideae e Tillandsioideae. ${ }^{1}$ As bromeliáceas são predominantemente neotropicais, com maior diversidade específica na floresta atlântica brasileira. Distribuem-se desde o Chile e Argentina, na América do Sul, Caribe e América Central, chegando a alcançar o México e o sudeste norte-americano. Apenas uma espécie, a Pitcarnia feliciana (A. Chevalier) Harms \& Mildbraed é encontrada fora do continente americano, na costa oeste africana. ${ }^{1}$

A descrição desta família é atribuída ao padre francês Charles Plumier que, no final do século XVII, se deparou com um conjunto de plantas diferentes e resolveu batizá-las de bromélias, em homenagem ao botânico sueco Olaf Bromel. Em 1789, foi estabelecida formalmente a reunião destas plantas, que se designou por Bromelie. ${ }^{2}$ Atualmente é considerada a segunda maior família de monocotiledôneas epífitas, sendo superada em número apenas pela família Orchidaceae. ${ }^{3}$

As bromeliáceas são notáveis pela sua diversidade ecológica, apresentando espécies terrestres e epífitas, adaptadas aos ambientes mésicos, semimésicos e xéricos. ${ }^{4}$ Podem ser encontradas desde o nível do mar até altitudes acima de $4000 \mathrm{~m}$. Há uma grande variedade de habitats, desde desérticos, quentes e secos, até florestas úmidas e regiões montanhosas mais frias. ${ }^{1,5}$ As espécies epífitas normalmente são encontradas sobre outras plantas, geralmente arbóreas, arbustivas ou cactáceas e, algumas vezes, sobre linhas de transmissão elétrica ou postes e telhados. ${ }^{5}$

As epífitas dessa família evoluíram de espécies terrestres para as matas úmidas e destas para o dossel, cuja atmosfera, servindo como fonte de água e nutrientes, permitiu que essas plantas se desligassem do solo. As adaptações que caracterizam tal evolução consistem na redução estrutural e funcional das raízes e na especialização dos tricomas foliares, escamas que podem suprir parcial ou totalmente a função de absorção das raízes. ${ }^{6}$

*e-mail: laverde@unipar.br
A família Bromeliaceae apresenta uma longa história de uso etnobotânico, associada aos povos americanos nativos, como fonte de fibras, alimentos, forragens e medicamentos, além de uso ornamental e místico. ${ }^{7}$ Estas categorias refletem, principalmente, antigas aplicações e percepções indígenas e nem sempre coincidem com seu uso moderno. ${ }^{1}$

Várias bromeliáceas apresentam potencial ornamental, o que vem causando o declínio das populações naturais de algumas espécies. Durante a descrição de uma das espécies descobertas por Morren, o mesmo escreveu sobre toda a família Bromeliaceae as seguintes linhas nas quais, segundo Pio Correa, ${ }^{8}$ seria inútil descobrir o mínimo de exagero:

... "Nós amamos, nas Bromeliaceas, o caracter, por assim dizer clássico, dos seus contornos: sua fórma é a de uma amphora, de um vaso grego ou de uma roseta; suas folhas descrevem curvas correctas, seus tecidos são firmes e sua superficie é frequentemente ornamentada com zebruras ou coloridos diversos; algumas defendem seus fructos com armas cerradas. As suas folhas são quasi sempre attrahentes $e$ sempre de longa duração, tem bellos enfeites e nada pediram à perfumaria”...

Muitas bromeliáceas ganharam popularidade entre paisagistas e jardineiros justamente devido à beleza de suas formas e cores, durabilidade das inflorescências, baixa demanda de cuidados e fácil adaptação a pequenos jardins. ${ }^{1,5}$ Incluem-se entre os gêneros frequentemente cultivados como ornamentais e conhecidos popularmente como bromélias, gravatás ou caraguatás: Aechmea, Alcantarae, Billbergia, Bromelia, Guzmania, Neoregelia, Pitcairnia, Tillandsia, Vriesia, entre outros. ${ }^{9}$

Apesar do nome desta família estar intimamente associado às bromélias, o abacaxi (Ananas comosus) é a espécie de Bromeliaceae mais conhecida, sendo inclusive considerado como uma das frutas tropicais mais populares do mundo. É o único representante desta 
família cultivado extensivamente como fonte de alimento. Ele também está fortemente associado à história dos aborígenes americanos, tanto como fonte de alimento quanto como elemento simbólico de rituais. ${ }^{7,10} \mathrm{O}$ abacaxi tornou-se muito popular principalmente pelo seu sabor e aroma marcantes. Seus frutos contêm a bromelina, uma enzima que os protege da depredação por larvas de insetos, a qual apresenta grande valor comercial e cujo uso se encontra em ascensão devido à sua aplicabilidade na indústria farmacêutica (atividade anti-helmíntica, anti-inflamatória e anticancerígena) ${ }^{11}$ e na indústria alimentícia (amaciante de carnes vermelhas, hidrolizante de complexos proteína-taninos na produção de cerveja, pães, leite de soja e ovos desidratados). ${ }^{12}$ Além da bromelina, muitas outras enzimas já foram isoladas e identificadas de diferentes bromeliáceas, as quais também apresentam grande potencialidade de ser empregada nestes mesmos segmentos industriais. ${ }^{13}$ Outras espécies de Ananas também apresentam potencial ornamental, sendo o nordeste brasileiro um grande exportador de abacaxi ornamental. ${ }^{14}$

Várias espécies do gênero Tillandsia são epífitas e adaptadas a ambientes secos. Possuem forma de crescimento altamente especializada, com escamas epidérmicas foliares capazes de absorver água e nutrientes diretamente da atmosfera. ${ }^{15}$ Esta capacidade de sobreviver em condições extremas está baseada em fatores morfológicos e fisiológicos. Nas espécies deste gênero, as raízes são reduzidas ou estão ausentes, apresentando apenas a função de fixação da planta ao substrato (árvores, fios de eletricidade, telhados, rochas etc), não desempenhando o papel absortivo, característico de plantas terrestres ou parasitas. Por esta razão, estas plantas são consideradas muito apropriadas para o monitoramento da poluição atmosférica, pois, juntamente com a água e os nutrientes, são capazes de acumularem poluentes e metais pesados presentes na atmosfera. Assim, a avaliação do conteúdo de seus tecidos reflete a contaminação atmosférica. ${ }^{16} \mathrm{Em}$ função disto, algumas espécies foram avaliadas como biomonitoras ambientais nas últimas décadas. Atualmente, a espécie Tillandsia usneoides é a mais empregada em estudos de biomonitoramento atmosférico de centros metropolitanos ${ }^{16}$ e regiões de garimpo, ${ }^{15}$ seguida das espécies T. recurvata, ${ }^{17,18}$ T. aeranthos, ${ }^{18}$ T. caput-medusae morren $^{19}$ e Tillandsia capillaris. ${ }^{20}$

Outro aspecto das bromeliáceas que pode ser assinalado é sua importância do ponto de vista econômico, pois suas folhas dão excelentes fibras. Algumas espécies de bromélias têm sido empregadas pelos americanos antigos como fontes de fibras desde antes da chegada de Colombo ao Novo Mundo. O curauá (Ananas erectifolius), espécie nativa amazônica, ganhou reconhecimento comercial como um material para acessórios na indústria automotiva brasileira. ${ }^{21} \mathrm{As}$ fibras desta espécie também têm sido estudadas como um material lignocelulósico alternativo para a produção de polpa de papel, com potencial aplicação na produção de papéis especiais. ${ }^{22}$

Considerando o grande número de espécies da família Bromeliaceae, poucas delas foram estudadas quimicamente até o momento. Apesar disto, há uma quantidade considerável de compostos identificados, os quais pertencem principalmente às classes dos triterpenoides e flavonoides. Outras classes de compostos como esteróis, diterpenos, ácidos cinâmicos, gliceróis substituídos, lignanas, compostos nitrogenados, entre outros, também foram identificados nesta família, embora em número bastante reduzido. Do ponto de vista farmacológico, também há poucos trabalhos descritos na literatura.

A presente revisão tem por objetivo sumarizar os principais aspectos químicos, farmacológicos ou biológicos de espécies desta família, indicando, quando possível, os compostos identificados de cada espécie, bem como a ação farmacológica ou biológica das bromeliáceas descritas na literatura. Espera-se que esta revisão possa representar um ponto de partida para futuras pesquisas enfocando a química e as atividades desta importante família da flora brasileira.

\section{ASPECTOS ETNOBOTÂNICOS, ATIVIDADES BIOLÓGICAS E FARMACOLÓGICAS}

O uso etnobotânico de algumas bromeliáceas está associado à história de vários grupos pré-colombianos, principalmente em usos medicinais, alimentares e místicos. ${ }^{7}$

Além de ser empregado como alimento, o abacaxi (Ananas comosus) também é bastante utilizado na medicina popular. Ele apresenta reconhecidas propriedades medicinais, agindo principalmente como estomáquico, carminativo, diurético e anti-inflamatório, sendo também indicado para problemas das vias respiratórias e para neurastenia. ${ }^{23} \mathrm{Na}$ Tailândia, é usado como planta medicinal no tratamento da disúria. Na China, o córtex serve como agente alexifármaco, antidiarreico e antitussivo, e as folhas são usualmente empregadas como agente contra a dispepsia e antidiarreico. ${ }^{24} \mathrm{Na}$ Índia, diz-se que o suco das folhas e frutos verdes apresenta atividade anti-helmíntica e propriedades abortivas. ${ }^{25}$ Estudos de extratos etanólicos das folhas de A. comosus mostraram efeitos antidiabético, anti-hiperlipidimêmico e antioxidante. ${ }^{26}$ Estudos recentes do mesmo grupo de pesquisa com o objetivo de investigar a sensibilidade à insulina em ratos diabéticos e células HepG2 foram otimistas. Os resultados sugerem que os extratos de $A$. comosus podem aumentar a sensibilidade à insulina em ratos com diabetes do tipo II. Além disso, os extratos inibiram o desenvolvimento de resistência à insulina em células de HepG2. ${ }^{24}$ Estudos estão sendo conduzidos para determinar o mecanismo hipolipidêmico destes extratos. ${ }^{27}$ Muitas outras atividades também são sugeridas para o abacaxi, como anti-inflamatória, antioxidante, depurativa, proteolítica, tônica etc. ${ }^{28}$

$\mathrm{O}$ Ananas sativus também é um excelente digestivo. O suco do fruto maduro é preconizado nas bronquites, tendo ainda ação benéfica sobre os intestinos e a acidez do estômago. O suco dos frutos verdes é vermífugo, abortivo, eficaz contra a retenção de urina e dores nos rins. ${ }^{8}$

O suco de Ananas muricata (Schult) constitui um energético diurético, indicado às doenças do aparelho urinário, em especial na hidropisia. Este mesmo suco é usado como expectorante e contra tosse e bronquite, acreditando-se ser um valioso remédio contra asma e coqueluche. ${ }^{29}$

No interior de Minas Gerais, o decocto ou o infuso dos frutos de Ananas microstachys Lind., popularmente conhecido como ananás, é usado como diurético e digestivo. ${ }^{30}$

Outra planta da subfamília Bromelioideae que também é bastante empregada na medicina tradicional brasileira é a Bromelia antiacantha Bertol., popularmente conhecida como caraguatá, carauatá, gravatá. Os frutos desta espécie são usados como emolientes, expectorantes em infecções respiratórias, contra asma, tosse e bronquite, ${ }^{31-33}$ e as folhas maceradas são antitérmicas e anti-helmínticas. ${ }^{31} \mathrm{O}$ decocto das folhas é utilizado em casos de problemas respiratórios e tosse. ${ }^{34}$ Além disso, no sul do Brasil, os frutos e folhas são empregados em casos de aftas e feridas. ${ }^{35}$

O decocto das raízes de macambira (Bromelia laciniosa) é usado popularmente contra hepatite e desarranjos intestinais, como diurético, enquanto que as folhas secas e pulverizadas são utilizadas na culinária como fonte de proteína. ${ }^{36}$ Os frutos da Bromelia plumieri, conhecida popularmente no Brasil como caroá ou como piñuela nos demais países latinoamericanos, são consumidos como vermífugos. ${ }^{36}$ Já os frutos de Bromelia fastuosa são usados em forma de xarope em casos de afecções asmáticas, bronquites e na opilação; também são considerados vermífugos, diuréticos e até abortivos. ${ }^{8}$ No Piauí, o chá de croatá ou coroatá (Bromelia karatas L.) é usado contra coqueluche e gripe. ${ }^{37}$

Os frutos de Bromelia pinguin, conhecidos como fruto maia, são consumidos em todo o Caribe, México, Panamá e Guiana. ${ }^{38}$ No México, estes frutos acídulos são reputados como vermífugos, diu- 
réticos e até abortivos. ${ }^{8}$ Os frutos desta espécie também são usados no tratamento de coqueluche, escorbuto, diabetes e doenças renais. ${ }^{39}$ Camacho-Hernandez e colaboradore ${ }^{39}$ avaliaram a atividade antifúngica de extratos metanólicos das polpas de B. pinguin contra várias cepas de fungos, revelando significativa atividade dos mesmos contra nove cepas de Trichophyton spp. e contra Paecillomyces variotii. Porém, foram insensíveis às cepas de Candida spp. Os extratos das folhas e ramos também têm mostrado atividades espasmódicas, antiparasitárias e citotóxicas. ${ }^{38,40,41} \mathrm{Na}$ Jamaica, as sementes de $B$. pinguin são usadas para a obtenção de corantes usados na culinária. ${ }^{8}$

A espécie Nidullarium procerum é uma das bromélias mais prevalentes no Brasil, habitando a floresta atlântica brasileira. Alguns estudos farmacológicos foram realizados com extratos aquosos das folhas desta espécie e revelaram que apresenta importante potencial terapêutico, com destaque particular à atividade antialérgica, uma vez que apresenta potente atividade antieosinófila. ${ }^{42}$ Estudos complementares mostraram que os extratos de $N$. procerum apresentam uma faixa de efeitos anti-inflamatórios distintos dos efeitos antialérgicos, incluindo potente atividade analgésica, ${ }^{43}$ a qual não está associada com a ativação de receptores opioides, mas à inibição seletiva da produção de $\mathrm{PGE}_{2}$. Os mecanismos envolvendo os efeitos antiinflamatórios do extrato aquoso desta espécie também foram avaliados sequencialmente pelo mesmo grupo de pesquisa. ${ }^{44}$

Na subfamília Tillandsioideae, a espécie mais estudada é a Tillandsia usneoides. Esta espécie é conhecida popularmente no Caribe, México e Estados Unidos como Spanish moss (musgo espanhol), enquanto no Brasil é conhecida como barba-de-velho ou barba-depau. No sul dos Estados Unidos, o chá feito desta espécie é usado para amenizar os sintomas de diabetes mellitus. ${ }^{45}$ Extratos aquosos e etanólicos de $T$. usneoides foram avaliados em ratos produzindo em um período de $24 \mathrm{~h}$ significante hipoglicemia. Estudos indicaram que o ácido 3-hidroxi-3-metilglutárico é o responsável pela ação hipoglicêmica, sendo sugerido como hipoglicemiante no tratamento de diabetes mellitus. ${ }^{46}$ Esta espécie apresenta ainda outras atividades como antirreumática, anti-hemorróide, antiespasmódico, diurética, anti-hipertensiva, colagoga, antibiótica, contra doenças renais e oftálmicas. ${ }^{8,32,46-48} \mathrm{~A}$ atividade antiviral de extratos diclorometano, acetato de etila e $n$-butanol das partes aéreas de T. usneoides foi investigada contra dois vírus, o herpes simples tipo 1 (HSV-1) e o poli vírus tipo 2 (PV-2). ${ }^{32}$ Segundo os autores deste estudo, os extratos mostraram inibição para o HSV-1, possivelmente devido aos teores de flavonoides presentes.

A espécie Tillandsia recurvata, também conhecida como barba-de-velho, é usada popularmente contra reumatismo, úlcera e hemorróide. ${ }^{36,48}$ Em Curaçao, no Caribe, o decocto das folhas é usado como emenagogo. ${ }^{49}$ No Uruguai, as partes aéreas são empregadas como antiespasmódico e para infecções dos olhos. ${ }^{50}$ No Equador, é usada no tratamento da vesícula, tosse, febre, dor de cabeça e dores no peito. Na Argentina, é usada no tratamento de hemorróidas. ${ }^{49}$ Os extratos das partes aéreas desta espécie foram ativos contra vários micro-organismos. ${ }^{50}$

A epífita $T$. streptocarpa, popularmente conhecida como gravatá-do-ar, é empregada como purgativo, laxativo, emético e analgésico. ${ }^{36} \mathrm{~A}$ infusão de suas partes aéreas também é usada como agente anti-inflamatório e contraceptivo.$^{51,52}$ Delaporte e colaborado$\mathrm{res}^{53}$ avaliaram a toxicidade aguda e atividades antiedematogênica, antioxidante e antimicrobiana de extratos metanólicos das partes aéreas desta espécie, sendo observadas expressiva atividade antiedematogênica e potente atividade antioxidante. Quanto à atividade antimicrobiana, os extratos se mostraram inativos frente aos microorganismos testados. Os extratos das partes aéreas apresentaram baixa toxicidade, suportando o uso tradicional desta espécie no tratamento de processos inflamatórios.
No Brasil, a espécie Tillandsia stricta é usada como diurético, a qual ainda apresenta um efeito antiblenorrágico. ${ }^{49}$ No Uruguai, as partes aéreas de T. aeranthos são usadas como antiespasmódico e para infecções dos olhos. Estudos mostraram que os extratos brutos desta espécie apresentam atividade antimicrobiana. ${ }^{50}$

Com relação ao uso etnobotânico e medicinal de espécies da subfamília Pitcairnioideae, não foi encontrado nenhum relato até o momento.

\section{A QUÍMICA DA FAMÍLIA BROMELIACEAE}

Algumas espécies de plantas da família Bromeliaceae foram e estão sendo estudadas química e farmacologicamente e, com isto, muitos compostos foram isolados e identificados. Diferentes classes de compostos orgânicos existentes nessas espécies foram relatadas, incluindo triterpenos, esteroides, flavonoides, derivados de ácidos cinâmicos, gliceróis, entre outros.

O Ananas comosus é a espécie mais estudada quimicamente nesta família, em função do valor comercial do seu suco, um dos mais consumidos no mundo. Desde a década de 1940, vários estudos foram realizados com o intuito de identificar as substâncias responsáveis pelo seu aroma. ${ }^{54}$ Mais de 280 constituintes voláteis foram encontrados desde então, porém poucos deles foram identificados como sendo contribuintes efetivos do aroma do abacaxi. Entre os constituintes chave do aroma do abacaxi, encontram-se a clássica furanona do abacaxi (4-hidroxi-2,5-dimetil$3[2 \mathrm{H}]$-furanona $(\mathrm{HDF})$ ou furaneol $\left.{ }^{\circledR}\right)$, ésteres como 2-metilbutanoatos de metila e etila, 2-metilpropanoato de etila, hexanoato de metila, 3-(metiltio)propanoatos de metila e etila, como também vários hidroxi e acetoxi ésteres, $\gamma$-lactonas e o 1-(E,Z)-3,5-undecatrieno. Considerando o elevado número de constituintes voláteis identificado, as estruturas dos mesmos não foram relatadas neste trabalho.

Na sequência, são apresentadas as estruturas das substâncias identificadas nas demais classes de compostos orgânicos encontrados em Bromeliaceae.

\section{Terpenoides}

A Tabela 1 apresenta todos os triterpenoides e esteroides isolados de plantas da família Bromeliaceae até o presente momento (Figura 1). Curiosamente, os triterpenoides isolados estão restritos às subfamílias Bromelioideae e Tillandsioideae, sendo mais abundantes nesta segunda subfamília. Dentre os triterpenoides isolados, foi constatada a presença de cicloartanos (1-9), lanostanos (10), labdanos (11-12), derivados do arborinol (13-15), a friedelina (16) e esteroides (17-25).

Uma grande variedade de cicloartanos foi encontrada na subfamília Tillandsioideae (Tabela 1). Embora, apenas 3 cicloartanos tenham sido isolados do Ananas comosus (subfamília Bromelioideae), o ácido ananásico $(\mathbf{4 d})^{57}$ e os ananasasídeos A (5d) e B $(\mathbf{6 d})^{55}$ apresentam um padrão de hidroxilação do esqueleto cicloartano (hidroxilas nas posições C-12, C-15 e C-16) que os diferem dos demais cicloartanos isolados das epífitas da subfamília Tillandsioideae. Curiosamente, a cadeia carboxílica lateral destes três compostos é idêntica.

Alguns cicloartanos apresentam cadeia lateral pouco convencional para triterpenoides e esteroides. Neste sentido, dois hidroperoxicicloartanos (1h, 2k) foram isolados das espécies Tillandsia usneoides ${ }^{64}$ e Tillandsia recurvata, ${ }^{63}$ provavelmente resultantes de um processo de foto-oxidação natural. Este processo foi sugerido para explicar a biogênese de alguns derivados de cicloartanos. ${ }^{70}$ Por se tratar de plantas expostas a condições adversas, como extrema exposição ao ar e à luz solar, ausência de umidade em função de suas características epífitas, os hidroperoxicicloartanos poderiam ser considerados como componentes do sistema protetor destas espécies. ${ }^{64}$ 
Tabela 1. Distribuição de triterpenoides e esteroides na família Bromeliaceae

Espécies

Ananas comosus

Ananas comosus var. Cayenne

Ananas erectofolius

Greigia sphacelata

Subfamília Pitcairnioideae

Hechtia rosea

Hechtia scariosa

Subfamília Tillandsioideae

Tillandsia brachycaulos

Tillandsia fasciculata

Tillandsia pohliana

Tillandsia recurvata

Tillandsia streptocarpa

Tillandsia usneoides isopropenil é bastante rara, presente em pouquíssimas espécies de plantas terrestres. Esta mesma cadeia também foi encontrada recentemente em dois labdanos (11r e 12r) isolados de T. brachycaulos $^{59}$ e T. pohliana, ${ }^{62}$ respectivamente (Figura 1). Poucos cicloartanos com cadeia lateral reduzida e 3,4-seco-cicloartanos são conhecidos na literatura. Os compostos $(\mathbf{1 x}),(\mathbf{2} \mathbf{s}),(\mathbf{2 u}),(\mathbf{2} \mathbf{v}),(\mathbf{2} \mathbf{w})$ e $(\mathbf{2} \mathbf{x})$, isolados de Tillandsia usneoides $^{64,67}$ apresentaram cadeia lateral (27-nor, 25,26,27-trinor, 24,25,26,27-tetranor) reduzida (Figura 1). Segundo os autores, ${ }^{68}$ a origem biogenética de cadeias reduzidas poderia estar relacionada a um processo de auto-oxidação in vivo.

Em relação aos esteróis isolados, dois deles apresentaram estruturas diferenciadas: o esterol (22p) e o peróxido ergosterol (24o)
(Figura 1). Segundo Pakrashi e colaboradores, ${ }^{25}$ o composto $(\mathbf{2 2 p})$ e seu epímero exibiram atividade antifertilidade significante em ratos.

Apenas quatro diterpenos (26-29) foram isolados de Bromeliaceae até o momento (Figura 2). Apesar do número bastante reduzido, dois deles, os filocladanos oxigenados (26) e (27), são raros na natureza e foram isolados de Bromelia pinguin. ${ }^{38}$ Segundo os autores, estes compostos são de considerável interesse, uma vez que cauranoides oxigenados exibem uma larga variedade de atividades fisiológicas e estes filocladanos poderiam apresentar atividades similares. Finalizando os compostos com esqueleto terpênico, a saponina (30) denominada de hecogenina, foi isolada da espécie Hechtia texensis. ${ }^{71}$

\section{Flavonoides}

A ocorrência de flavonoides na família Bromeliaceae permite destacar a importância química dos mesmos como possíveis agentes farmacológicos e, também, considerá-los como potenciais marcadores quimiotaxonômicos. Foram identificados até o momento 2 diferentes flavanóis (31; Figura 3), 3 flavanonas (32; Figura 3), 26 flavonas (33-34; Figura 4), 25 flovonóis (35-38; Figura 5) e 20 antocianinas (39; Figura 6) em 83 espécies de Bromeliaceae avaliadas, sendo 30 da subfamília Bromelioideae, 26 da subfamília Pitcairnioideae e 27 da subfamília Tillandsioideae. A ocorrência dos flavonoides nas três subfamílias de Bromeliaceae foi sumarizada na Tabela 2. As maiores contribuições foram realizadas por Willians, ${ }^{72}$ com a identificação de flavonas e flavonóis em cerca de 37 espécies, por Satto e Harborne ${ }^{73}$ com a identificação de antocianinas em cerca de 34 espécies e por Scogin, ${ }^{74}$ com a identificação de antocianinas em 9 espécies dos gêneros Puya e Puyopsis.

Embora várias classes de flavonoides sejam representadas na família Bromeliaceae, os constituintes mais distintos são aqueles que apresentam hidroxilação ou metoxilação na posição C-6. Sem dúvida, a predominância destes compostos nesta família é única dentro das monocotiledônias. ${ }^{72}$ A ocorrência de um padrão particular de substituição é frequentemente usada como um indicador de avanço filogenético. Quimicamente, 6- ou 8-hidroxi ou metoxiflavonoides são considerados como caracteres avançados, resultado de uma etapa biossintética extra para sua formação.

Analisando a relação dos flavonoides identificados em Bromeliaceae até o momento, cerca de $40 \%$ apresentam as posições C- 6 ou C-8 oxigenadas. Todas as flavanonas são metoxiladas nesta posição e pouco mais da metade das flavonas são oxigenadas em C-6, com uma relação hidroxilação:metoxilação de cerca de 4:3. Aproximadamente a metade dos flavonóis é oxigenada em C-6 (100\% metoxilação) e nenhuma antocianina apresenta oxigenação nesta posição específica. Considerando a ocorrência apenas de flavanonas, flavonas e flavonóis nas distintas subfamílias de Bromeliaceae (Tabela 3), verificou-se que a incidência destes compostos (oxigenação em C-6) na subfamília Bromelioideae corresponde a menos de um quinto (18\%) das substâncias. Na subfamília Pitcairnioideae, metade das ocorrências correspondem exclusivamente a flavonas C-6 oxigenadas, sendo a maioria hidroxilada (apenas $13 \%$ é metoxilada). $\mathrm{Na}$ subfamília Tillandsioideae, a ocorrência destes compostos com oxigenação em C-6 corresponde ao dobro de entradas em relação aos não oxigenados em C-6, sendo que a maioria (96\%) apresenta metoxilação nesta posição específica.

Com base nesta distribuição (Tabela 3), poder-se-ia avaliar o grau de evolução das três subfamílias de Bromeliaceae quimiotaxonomicamente. A subfamília Bromelioideae apareceria como a mais primitiva, seguida da subfamília Pitcairnioideae e a mais evoluída seria a subfamília Tillandsioideae. Taxonômica e anatomicamente, a subfamília Tillandsioideae é vista como a mais evoluída dentro da 


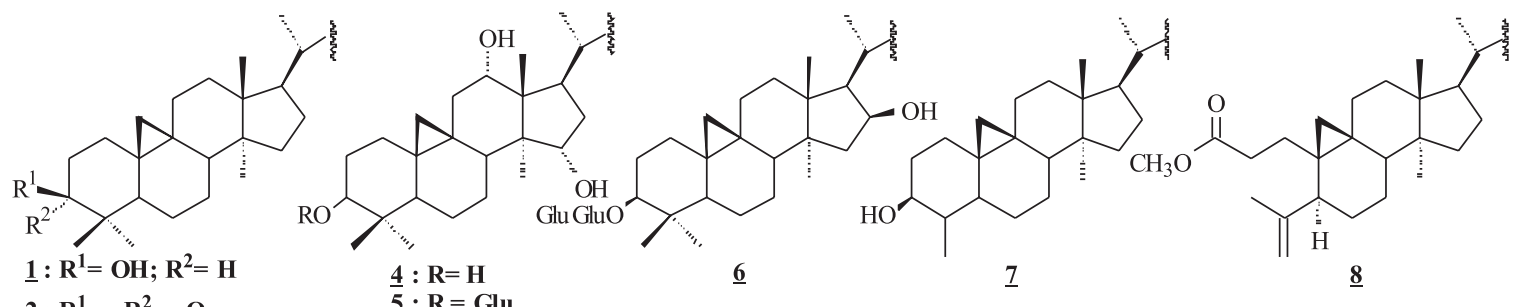

$$
\begin{aligned}
& \underline{2}: R^{1}=R^{2}=O \\
& \underline{3}: R^{1}=\text { OCHO; } R^{2}=H
\end{aligned}
$$$$
\underline{\mathbf{5}}: \mathbf{R}=\mathbf{G l u}
$$
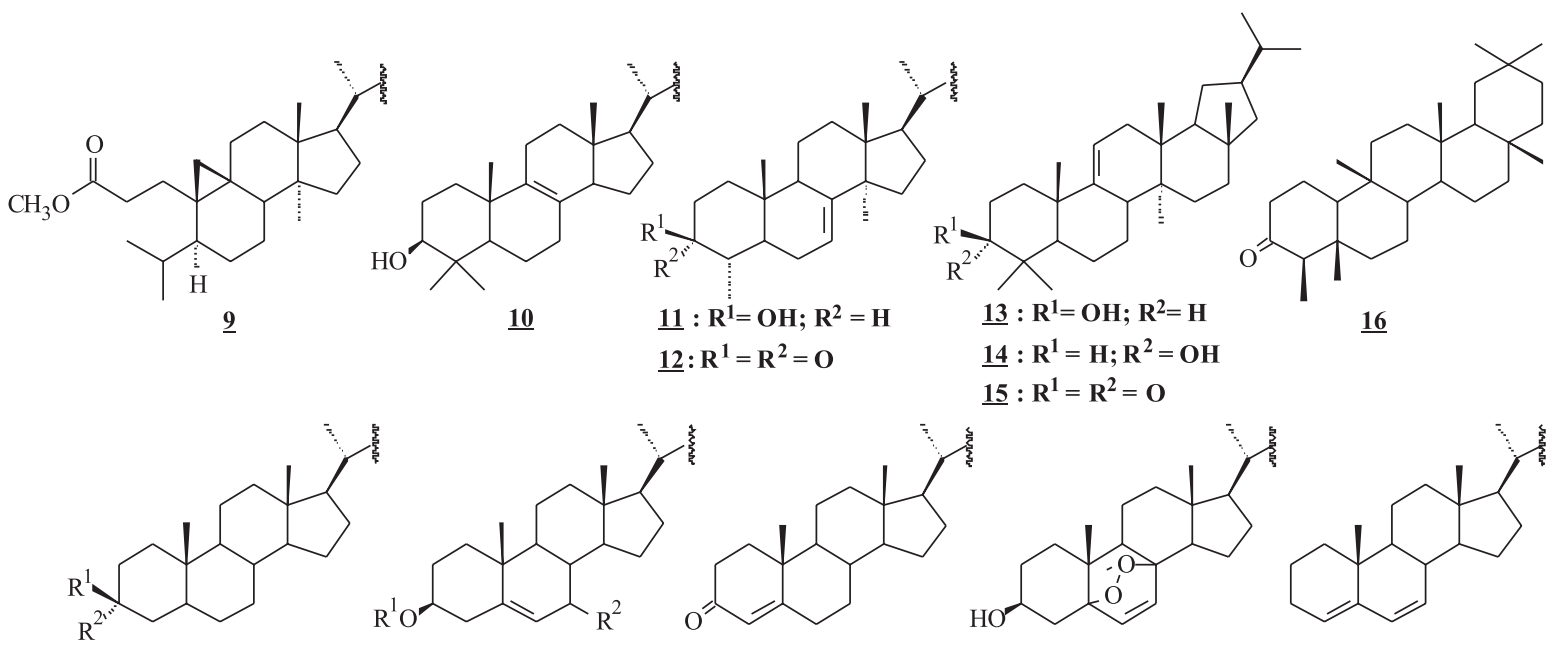

17: $\mathbf{R}^{1}=\mathbf{R}^{2}=\mathbf{H}$

$\underline{20}: \mathbf{R}^{1}=\mathbf{R}^{2}=\mathbf{H}$

23

$15: R^{1}=R^{2}=0$

18: $\mathbf{R}^{1}=\mathbf{R}^{2}=\mathbf{O}$

$\underline{21}: \mathrm{R}^{1}=\mathrm{Glu} ; \mathrm{R}^{2}=\mathrm{H}$

19: $R^{1}=O H ; R^{2}=H$

$22: \mathbf{R}^{1}=\mathbf{H} ; \mathbf{R}^{2}=\mathrm{OH}$

(N)<smiles>C=C(C)C(O)CCCC(O)C(=C)C</smiles><smiles>CC(C)(O)C=CCC(C)(C)C</smiles>

$\mathrm{O}_{\mathrm{OCH}}$<smiles>CCC=CC(CCCC)C(C)C</smiles><smiles>CCCC(C)C(C)C(C)C</smiles><smiles>C=C(CCI)C(C)C</smiles><smiles>C=C(C)C(C)CCI</smiles><smiles>CC=CC(C)C(C)C</smiles><smiles>CCCC(CC)C(C)C</smiles>

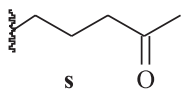<smiles>CCCC=CC(C)=O</smiles><smiles>CCCCO</smiles><smiles>CCCCC=O</smiles><smiles>[13CH3]/C=C/C=O</smiles><smiles>[CH]C=[CH]</smiles>

Figura 1. Triterpenoides e esteroides presentes em Bromeliaceae. As diferentes cadeias laterais estão representadas por letras (a-x)<smiles>C=CC1(C)CCC2C(C1)C(O)CC1C(C)(C)C(=O)CCC21C</smiles><smiles>C=CC1(C)CCC2C3CCCC(C)(C)C3CCC2C1(C)C(=O)O</smiles><smiles>CC1(O)CC23CCC4C(C)(CO)CCCC4(C)C2CCC1C3</smiles><smiles>CC1(C)CCC23CCC4C(C)(C)C(=O)CCC4(C)C2CCC1C3</smiles><smiles>CC1CCC2(OC1)OC1C3CCC4CC(O)CCC4(C)C3CC(=O)C12C</smiles>

$\underline{30}$<smiles>[Y]c1cc(O)cc(C2CC(=O)c3c(O)c([R])c([Y])c([R])c3OC2c2cc([Z7])c([Y7])c([Y])c2)c1</smiles>

31 $\underline{\text { a }}:-\mathrm{OH}$

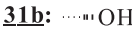

32 2 a $: R^{1}=R^{5}=R^{6}=O H_{3} ; R^{2}=R^{4}=O H ; R^{3}=H$ $\underline{32 b}: \mathbf{R}^{1}=\mathbf{R}^{2}=\mathbf{R}^{5}=\mathbf{R}^{6}=\mathbf{O C H}_{3} ; \mathbf{R}^{4}=\mathrm{OH} ; \mathbf{R}^{3}=\mathbf{H}$ 32C : $\mathbf{R}^{1}=\mathbf{R}^{2}=R^{3}=R^{5}=O_{C H} ; R^{4}=O H ; R^{6}=H$
Figura 3. Flavanois catequina (31a) e epicatequina (31b) e flavanonas (32a-c) relatados na família Bromeliaceae 
<smiles>[R]c1cc(-c2cc(=O)c3c(O)c([R])c([R])cc3o2)cc([R])c1[R]</smiles>

$\underline{3} \underline{3}$<smiles>O=c1cc(-c2ccc(O)cc2)oc2cc(O)c([C@@H]3O[C@H](CO)[C@@H](O)[C@H](O)C3O)c(O)c12</smiles>

\begin{tabular}{|c|c|c|c|c|c|c|}
\hline Flavona & 33 & $\mathbf{R}^{1}$ & $\mathbf{R}^{2}$ & $\mathbf{R}^{3}$ & $\mathbf{R}^{4}$ & $\mathbf{R}^{5}$ \\
\hline Apigenina & a & $\mathrm{H}$ & $\mathrm{OH}$ & $\mathrm{H}$ & $\mathrm{OH}$ & $\mathrm{H}$ \\
\hline Luteolina & b & $\mathrm{H}$ & $\mathrm{OH}$ & $\mathrm{OH}$ & $\mathrm{OH}$ & $\mathrm{H}$ \\
\hline Tricina & c & $\mathrm{H}$ & $\mathrm{OH}$ & $\mathrm{OCH}_{3}$ & $\mathrm{OH}$ & $\mathrm{OCH}_{3}$ \\
\hline 7-O-glicopiranosil-apigenina & d & $\mathrm{H}$ & $O$-Glu & $\mathrm{H}$ & $\mathrm{OH}$ & $\mathrm{H}$ \\
\hline Apiina & e & $\mathrm{H}$ & $O$-apioglu & $\mathrm{H}$ & $\mathrm{OH}$ & $\mathrm{H}$ \\
\hline 7-O-diglucorinosil-apigenina & f & $\mathrm{H}$ & $O$-diglucor & $\mathrm{H}$ & $\mathrm{OH}$ & $\mathrm{H}$ \\
\hline 7-O-apiosilglucosil-luteolina & g & $\mathrm{H}$ & $O$-apioglu & $\mathrm{OH}$ & $\mathrm{OH}$ & $\mathrm{H}$ \\
\hline 7-O-glicopiranosil-luteolina & $\mathbf{h}$ & $\mathrm{H}$ & $O$-Glu & $\mathrm{OH}$ & $\mathrm{OH}$ & $\mathrm{H}$ \\
\hline 7- $O$-rhamnopiranosil-luteolina & $\mathbf{i}$ & $\mathrm{H}$ & $O$-Rha & $\mathrm{OH}$ & $\mathrm{OH}$ & $\mathrm{H}$ \\
\hline 7-O-rutinosil-luteolina & $\mathbf{j}$ & $\mathrm{H}$ & $O-\mathrm{Ru}$ & $\mathrm{OH}$ & $\mathrm{OH}$ & $\mathrm{H}$ \\
\hline Ananaflavosídeo A & $\mathbf{k}$ & $\mathrm{H}$ & $\mathrm{OCH}_{3}$ & $\mathrm{OCH}_{3}$ & $O$-Glu & $\mathrm{OCH}_{3}$ \\
\hline Scutelareína & $\mathbf{L}$ & $\mathrm{OH}$ & $\mathrm{OH}$ & $\mathrm{H}$ & $\mathrm{OH}$ & $\mathrm{H}$ \\
\hline 7-O-rutinosil-scutelareína & $\mathbf{m}$ & $\mathrm{OH}$ & $O-\mathrm{Ru}$ & $\mathrm{H}$ & $\mathrm{OH}$ & $\mathrm{H}$ \\
\hline 6-OH-luteolina & n & $\mathrm{OH}$ & $\mathrm{OH}$ & $\mathrm{OH}$ & $\mathrm{OH}$ & $\mathrm{H}$ \\
\hline 7-O-glicopiranosil-6-OH-luteolina & $\mathbf{o}$ & $\mathrm{OH}$ & $O$-Glu & $\mathrm{OH}$ & $\mathrm{OH}$ & $\mathrm{H}$ \\
\hline 7-O-rhamnopiranosil-6-OH-luteolina & $\mathbf{p}$ & $\mathrm{OH}$ & $O$-Rha & $\mathrm{OH}$ & $\mathrm{OH}$ & $\mathrm{H}$ \\
\hline 7-O-rutinosil-6-OH-luteolina & q & $\mathrm{OH}$ & $O$-Rut & $\mathrm{OH}$ & $\mathrm{OH}$ & $\mathrm{H}$ \\
\hline Ananaflavosídeo C & $\mathbf{r}$ & $\mathrm{OH}$ & $\mathrm{OCH}_{3}$ & $\mathrm{OCH}_{3}$ & $O$-Glu & $\mathrm{OCH}_{3}$ \\
\hline Ananaflavosídeo D & $\mathbf{s}$ & $O$-Glu & $\mathrm{OCH}_{3}$ & $\mathrm{OCH}_{3}$ & $\mathrm{OH}$ & $\mathrm{OCH}_{3}$ \\
\hline Cirsimaritina & $\mathbf{t}$ & $\mathrm{OCH}_{3}$ & $\mathrm{OCH}_{3}$ & $\mathrm{H}$ & $\mathrm{OH}$ & $\mathrm{H}$ \\
\hline Jaceosidina & $\mathbf{u}$ & $\mathrm{OCH}_{3}$ & $\mathrm{OH}$ & $\mathrm{OCH}_{3}$ & $\mathrm{OH}$ & $\mathrm{H}$ \\
\hline 6,7-dimetoxi-5, 3',4'-triidroxiflavona & $\mathbf{v}$ & $\mathrm{OCH}_{3}$ & $\mathrm{OCH}_{3}$ & $\mathrm{OH}$ & $\mathrm{OH}$ & $\mathrm{H}$ \\
\hline Cirsilineol & w & $\mathrm{OCH}_{3}$ & $\mathrm{OCH}_{3}$ & $\mathrm{OCH}_{3}$ & $\mathrm{OH}$ & $\mathrm{H}$ \\
\hline $\begin{array}{l}\text { 4'-O-glicopiranosil-6,7,3'-trimetoxi-5,4'- } \\
\text { diidroxiflavona }\end{array}$ & $\mathbf{x}$ & $\mathrm{OCH}_{3}$ & $\mathrm{OCH}_{3}$ & $\mathrm{OCH}_{3}$ & $O$-Glu & $\mathrm{H}$ \\
\hline Ananaflavosídeo B & $\mathbf{y}$ & $\mathrm{OCH}_{3}$ & $\mathrm{OCH}_{3}$ & $\mathrm{OCH}_{3}$ & $O$-Glu & $\mathrm{OCH}_{3}$ \\
\hline Isovitexina & 34 & C-Glu & $\mathrm{OH}$ & $\mathrm{H}$ & $\mathrm{OH}$ & $\mathrm{H}$ \\
\hline
\end{tabular}

Figura 4. Flavonas relatadas na família Bromeliaceae

família Bromeliaceae, contudo, ainda há muitas incertezas quanto à mais primitiva. ${ }^{71}$ Embora nenhuma antocianina com padrão de oxigenação nas posições C-6 ou C-8 tenha sido identificada, também é observado certo grau de complexidade estrutural nesta classe de compostos devido à presença de cianidinas 3,5,3'-triglicosiladas $(39 q \text { e 39r })^{73}$ e delfinidinas 3,7,3'-triglicosiladas (39k). ${ }^{74}$ Além disso, também foram identificados outros flavonoides menos comuns, como é o caso da isovitexina (34), fisetina (36), morina (37) e gossipetina (38), cuja distribuição na família Bromeliaceae está restrita a pouquíssimas espécies.

\section{Ácidos cinâmicos e derivados}

Vários derivados do ácido cinâmico $(\mathbf{4 0 a - g} ; \mathbf{4 1})$ foram identificados em algumas espécies de Bromeliaceae ${ }^{27,38,49,56,58,76,77,81,87}$ (Figura 7). No final da década de 1950 , Sutherland e Gortner ${ }^{88}$ realizaram um estudo do tecido do caule do abacaxi, o qual revelou uma forma combinada de ácidos cinâmicos, um di-p-cumarato de quinila (42), o qual atua como um modificador da oxidase IAA do abacaxi. Outros dois ésteres do ácido quínico foram identificados em Ananas comosus (43-44) $)^{77}$ e Tillandsia brachycaulos (44) ${ }^{60}$ 
<smiles>[R]c1cc(-c2oc3cc([R])c([R])c(O)c3c(=O)c2[R])cc([R])c1[R]</smiles><smiles>O=c1c(O)c(-c2ccc(O)cc2O)oc2cc(O)cc(O)c12</smiles><smiles>O=c1c(O)c(-c2ccc(O)c(O)c2)oc2c(O)c(O)cc(O)c12</smiles>

\begin{tabular}{|c|c|c|c|c|c|c|c|}
\hline Flavonol & 35 & $\mathbf{R}$ & $\mathbf{R}^{1}$ & $\mathbf{R}^{2}$ & $\mathbf{R}^{3}$ & $\mathbf{R}^{4}$ & $\mathbf{R}^{5}$ \\
\hline Kaempferol & $\mathbf{a}$ & $\mathrm{OH}$ & $\mathrm{H}$ & $\mathrm{OH}$ & $\mathrm{H}$ & $\mathrm{OH}$ & $\mathrm{H}$ \\
\hline Quercetina & b & $\mathrm{OH}$ & $\mathrm{H}$ & $\mathrm{OH}$ & $\mathrm{OH}$ & $\mathrm{OH}$ & $\mathrm{H}$ \\
\hline Miricetina & c & $\mathrm{OH}$ & $\mathrm{H}$ & $\mathrm{OH}$ & $\mathrm{OH}$ & $\mathrm{OH}$ & $\mathrm{OH}$ \\
\hline Isorhamnetina & d & $\mathrm{OH}$ & $\mathrm{H}$ & $\mathrm{OH}$ & $\mathrm{OCH}_{3}$ & $\mathrm{OH}$ & $\mathrm{H}$ \\
\hline 3-O-rutinosil-kaempferol & e & $O$-Rut & $\mathrm{H}$ & $\mathrm{OH}$ & $\mathrm{H}$ & $\mathrm{OH}$ & $\mathrm{H}$ \\
\hline 3-O-glicopiranosil-quercetina & f & $O$-Glu & $\mathrm{H}$ & $\mathrm{OH}$ & $\mathrm{OH}$ & $\mathrm{OH}$ & $\mathrm{H}$ \\
\hline 3-O-rutinosil-quercetina & g & $O$-Rut & $\mathrm{H}$ & $\mathrm{OH}$ & $\mathrm{OH}$ & $\mathrm{OH}$ & $\mathrm{H}$ \\
\hline 3-O-glicopiranosil-myricetina & $\mathbf{h}$ & $O$-Glu & $\mathrm{H}$ & $\mathrm{OH}$ & $\mathrm{OH}$ & $\mathrm{OH}$ & $\mathrm{OH}$ \\
\hline 3-O-galactopiranosil-myricetina & i & $O-\mathrm{Gal}$ & $\mathrm{H}$ & $\mathrm{OH}$ & $\mathrm{OH}$ & $\mathrm{OH}$ & $\mathrm{OH}$ \\
\hline Tetrametil-éter myricetina & $\mathbf{j}$ & $\mathrm{OCH}_{3}$ & $\mathrm{H}$ & $\mathrm{OCH}_{3}$ & $\mathrm{OH}$ & $\mathrm{OCH}_{3}$ & $\mathrm{OCH}_{3}$ \\
\hline Retusina & $\mathbf{k}$ & $\mathrm{OCH}_{3}$ & $\mathrm{H}$ & $\mathrm{OCH}_{3}$ & $\mathrm{OCH}_{3}$ & $\mathrm{OCH}_{3}$ & $\mathrm{H}$ \\
\hline Patuletina & $\mathbf{L}$ & $\mathrm{OH}$ & $\mathrm{OCH}_{3}$ & $\mathrm{OH}$ & $\mathrm{OH}$ & $\mathrm{OH}$ & $\mathrm{H}$ \\
\hline $\begin{array}{l}6,3^{\prime}, 5^{\prime} \text {-trimetoxi-3,5,7,4'-tetraidroxifla- } \\
\text { vona }\end{array}$ & m & $\mathrm{OH}$ & $\mathrm{OCH}_{3}$ & $\mathrm{OH}$ & $\mathrm{OCH}_{3}$ & $\mathrm{OH}$ & $\mathrm{OCH}_{3}$ \\
\hline 3-O-glicopiranosil-patuletina & $\mathbf{n}$ & $O$-Glu & $\mathrm{OCH}_{3}$ & $\mathrm{OH}$ & $\mathrm{OH}$ & $\mathrm{OH}$ & $\mathrm{H}$ \\
\hline 3-O-rhamnopiranosil-patuletina & $\mathbf{o}$ & $O$-Rha & $\mathrm{OCH}_{3}$ & $\mathrm{OH}$ & $\mathrm{OH}$ & $\mathrm{OH}$ & $\mathrm{H}$ \\
\hline $\begin{array}{l}\text { 3-O-glicopiranosil-6,3',5' -trimetoxi- } \\
\text { 3,5,7,4'-tetraidroxiflavona }\end{array}$ & $\mathbf{p}$ & $O$-Glu & $\mathrm{OCH}_{3}$ & $\mathrm{OH}$ & $\mathrm{OCH}_{3}$ & $\mathrm{OH}$ & $\mathrm{OCH}_{3}$ \\
\hline $\begin{array}{l}3,6,3^{\prime}, 5^{\prime} \text {-tetrametoxi-5,7,4'-triidroxifla- } \\
\text { vona }\end{array}$ & $\mathbf{q}$ & $\mathrm{OCH}_{3}$ & $\mathrm{OCH}_{3}$ & $\mathrm{OH}$ & $\mathrm{OCH}_{3}$ & $\mathrm{OH}$ & $\mathrm{OCH}_{3}$ \\
\hline $\begin{array}{l}\text { 7-O-glicopiranosil-3,6,3',5' -tetrametoxi- } \\
\text { 5,7,4'-triidroxiflavona }\end{array}$ & $\mathbf{r}$ & $\mathrm{OCH}_{3}$ & $\mathrm{OCH}_{3}$ & $O$-Glu & $\mathrm{OCH}_{3}$ & $\mathrm{OH}$ & $\mathrm{OCH}_{3}$ \\
\hline Penduletina & $\mathbf{s}$ & $\mathrm{OCH}_{3}$ & $\mathrm{OCH}_{3}$ & $\mathrm{OCH}_{3}$ & $\mathrm{H}$ & $\mathrm{OH}$ & $\mathrm{H}$ \\
\hline 4'-metiléter penduletina & $\mathbf{t}$ & $\mathrm{OCH}_{3}$ & $\mathrm{OCH}_{3}$ & $\mathrm{OCH}_{3}$ & $\mathrm{H}$ & $\mathrm{OCH}_{3}$ & $\mathrm{H}$ \\
\hline Casticina & $\mathbf{u}$ & $\mathrm{OCH}_{3}$ & $\mathrm{OCH}_{3}$ & $\mathrm{OCH}_{3}$ & $\mathrm{OH}$ & $\mathrm{OCH}_{3}$ & $\mathrm{H}$ \\
\hline Artemetina & $\mathbf{v}$ & $\mathrm{OCH}_{3}$ & $\mathrm{OCH}_{3}$ & $\mathrm{OCH}_{3}$ & $\mathrm{OCH}_{3}$ & $\mathrm{OCH}_{3}$ & $\mathrm{H}$ \\
\hline
\end{tabular}

Figura 5. Flavonois relatados na família Bromeliaceae

Os ácidos hidroxicinâmicos e seus ésteres do ácido quínico são bastante comuns em todo o reino vegetal, sendo encontrados em quase todas as frutas e vegetais. Entretanto, 1,2- e 1,3-diglicerídeos naturais destes ácidos são pouco comuns, restritos a apenas algumas espécies das famílias Gramineae ${ }^{89}$ Liliaceae,${ }^{90}$ Sparganiaceae ${ }^{91}$ e do microorganismo Epicholoe typhina.$^{92}$ Em Bromeliaceae, estes diglicerídeos fenilpropanatos (Figura 8) foram identificados nas folhas de Ananas comosus $(\mathbf{4 5 c - h}),{ }^{56,77,93,94}$ Greigia sphacelata $(\mathbf{4 5 b}),{ }^{58}$ Tillandsia recurvata $(\mathbf{4 5 a})^{49}$ e Tillandsia streptocarpa $(\mathbf{4 5 c} \mathbf{c}-\mathbf{f}) \cdot{ }^{94}$ Futuros estudos sobre a distribuição destes diglicerídeos na família Bromeliaceae poderiam eventualmente contribuir para estudos quimiotaxonômicos. Alguns monoglicerídeos de ácidos cinâmicos também foram isolados de Ananas comosus (46a-b; 47a-b) $)^{27,56,77,88}$ e Greigia sphacelata (46a; 48a-b). ${ }^{58}$

\section{Outros compostos}

Estudo recente de Peñalvo e colaboradores ${ }^{96}$ identificou 6 lignanas dietéticas (Figura 9) nos frutos de Ananas comosus: pinoresinol (49), medioresinol (50), siringaresinol (51), lariciresinol (52), secoisolariciresinol (53) e matairesinol (54).

Ainda outros compostos fenólicos (Figura 10), como os ácidos gálico $(\mathbf{5 5})^{76}$ e tânico (56), ${ }^{81}$ foram identificados em Ananas comosus. Na Bromelia plumieri foi isolado o 3,4-dimetoxifenil- $\beta$-Dglucopiranosídeo (57). ${ }^{97}$

A presença de compostos nitrogenados em espécies da família Bromeliaceae é bastante modesta (Figura 11). Análises do suco dos frutos de abacaxi por HPLC revelaram a presença de alguns alcaloides do tipo tetraidro- $\beta$-carboniloide (58a-d).$^{98}$ Adrien-Romero e Blunden, ${ }^{99}$ através de um extenso trabalho, avaliaram a distribuição de betaínas nas partes aéreas de 38 espécies de bromeliáceas. Praticamente todas as espécies avaliadas apresentaram concentrações baixíssimas de glicinobetaína (59) e trigonelina (60), indicando certa homogeneidade na família. Segundo os autores, estas substâncias desempenham importante papel na adaptação de plantas a habitats 
<smiles>[R]c1cc([R])c2cc(-c3cc([R])c([R])c([R])c3)oc2c1</smiles>

\begin{tabular}{|c|c|c|c|c|c|c|c|}
\hline Antocianina & 39 & $\mathbf{R}$ & $\mathbf{R}^{1}$ & $\mathbf{R}^{2}$ & $\mathbf{R}^{3}$ & $\mathbf{R}^{4}$ & $\mathbf{R}^{5}$ \\
\hline Pelargonidina & a & $\mathrm{OH}$ & $\mathrm{OH}$ & $\mathrm{OH}$ & $\mathrm{H}$ & $\mathrm{OH}$ & $\mathrm{H}$ \\
\hline Cianidina & b & $\mathrm{OH}$ & $\mathrm{OH}$ & $\mathrm{OH}$ & $\mathrm{OH}$ & $\mathrm{OH}$ & $\mathrm{H}$ \\
\hline Peonidina & c & $\mathrm{OH}$ & $\mathrm{OH}$ & $\mathrm{OH}$ & $\mathrm{OCH}_{3}$ & $\mathrm{OH}$ & $\mathrm{H}$ \\
\hline Delfinidina & d & $\mathrm{OH}$ & $\mathrm{OH}$ & $\mathrm{OH}$ & $\mathrm{OH}$ & $\mathrm{OH}$ & $\mathrm{OH}$ \\
\hline 3-O-rutinosil-pelargonidina & e & $O$-Rut & $\mathrm{OH}$ & $\mathrm{OH}$ & $\mathrm{H}$ & $\mathrm{OH}$ & $\mathrm{H}$ \\
\hline 3-O-glicopiranosil-cianidina & f & $O$-Glu & $\mathrm{OH}$ & $\mathrm{OH}$ & $\mathrm{OH}$ & $\mathrm{OH}$ & $\mathrm{H}$ \\
\hline 3,3'-O-diglicopiranosil-cianidina & g & $O$-Glu & $\mathrm{OH}$ & $\mathrm{OH}$ & $O$-Glu & $\mathrm{OH}$ & $\mathrm{H}$ \\
\hline 3-O-rutinosil-3'-O-glicopiranosil-cianidina & $\mathbf{h}$ & $O$-Rut & $\mathrm{OH}$ & $\mathrm{OH}$ & $O$-Glu & $\mathrm{OH}$ & $\mathrm{H}$ \\
\hline 3-O-glicopiranosil-peonidina & $\mathbf{i}$ & $O$-Glu & $\mathrm{OH}$ & $\mathrm{OH}$ & $\mathrm{OCH}_{3}$ & $\mathrm{OH}$ & $\mathrm{H}$ \\
\hline 3-O-glicopiranosil-delfinidina & $\mathbf{j}$ & $O$-Glu & $\mathrm{OH}$ & $\mathrm{OH}$ & $\mathrm{OH}$ & $\mathrm{OH}$ & $\mathrm{OH}$ \\
\hline 3,7,3'-O-triglicopiranosil-delfinidina & $\mathbf{k}$ & $O$-Glu & $\mathrm{OH}$ & $O$-Glu & $O$-Glu & $\mathrm{OH}$ & $\mathrm{OH}$ \\
\hline 3-O-glicopiranosil-malvidina & $\mathbf{L}$ & $O$-Glu & $\mathrm{OH}$ & $\mathrm{OH}$ & $\mathrm{OCH}_{3}$ & $\mathrm{OH}$ & $\mathrm{OCH}_{3}$ \\
\hline 3,5-O-diglicopiranosil-pelargonidina & $\mathbf{m}$ & $O$-Glu & $O$-Glu & $\mathrm{OH}$ & $\mathrm{H}$ & $\mathrm{OH}$ & $\mathrm{H}$ \\
\hline 3-O-rutinosil-5-O-glicopiranosil-pelargonidina & $\mathbf{n}$ & $O$-Rut & $O$-Glu & $\mathrm{OH}$ & $\mathrm{H}$ & $\mathrm{OH}$ & $\mathrm{H}$ \\
\hline 3,5-O-diglicopiranosil-cianidina & $\mathbf{o}$ & $O$-Glu & $O$-Glu & $\mathrm{OH}$ & $\mathrm{OH}$ & $\mathrm{OH}$ & $\mathrm{H}$ \\
\hline 3,5-O-diglicopiranosil-delfinidina & $\mathbf{p}$ & $O$-Glu & $O$-Glu & $\mathrm{OH}$ & $\mathrm{OH}$ & $\mathrm{OH}$ & $\mathrm{OH}$ \\
\hline 3,5,3'-O-triglicopiranosil-cianidina & $\mathbf{q}$ & $O$-Glu & $O$-Glu & $\mathrm{OH}$ & $O$-Glu & $\mathrm{OH}$ & $\mathrm{H}$ \\
\hline 3-O-rutinosil-5,3'-O-diglicopiranosil-cianidina & $\mathbf{r}$ & $O$-Rut & $O$-Glu & $\mathrm{OH}$ & $O$-Glu & $\mathrm{OH}$ & $\mathrm{H}$ \\
\hline 3,5-O-diglicopiranosil-peonidina & $\mathbf{s}$ & $O$-Glu & $O$-Glu & $\mathrm{OH}$ & $\mathrm{OCH}_{3}$ & $\mathrm{OH}$ & $\mathrm{H}$ \\
\hline 3,5-O-diglicopiranosil-malvidina & $\mathbf{t}$ & $O$-Glu & $O$-Glu & $\mathrm{OH}$ & $\mathrm{OCH}_{3}$ & $\mathrm{OH}$ & $\mathrm{OCH}_{3}$ \\
\hline
\end{tabular}

Figura 6. Antocianinas relatadas na família Bromeliaceae

salinos e secos, resistência ao ataque de patógenos etc.

Estudos sobre precursores flavorizantes de frutas tropicais resultaram no isolamento do antranilato de $1-O-\beta$-glucopiranosila $(\mathbf{6 1})$ dos frutos de piñuela (Bromelia plumieri). ${ }^{100}$

Alguns $S$-derivados (62-64) do álcool sinapílico foram isolados do suco de abacaxi. ${ }^{101}$ Tais compostos são pouco comuns na natureza.

Marques e colaboradores ${ }^{22}$ avaliaram recentemente o extrato apolar das fibras do Ananas erectifolius e identificaram uma série de ácidos graxos, alcoóis graxos, $\alpha$ - e $\omega$-hidroxi-ácidos graxos, monoglicerídeos e graxas, todos de cadeia longa. Outros compostos como $\omega$-hidroxi-monoésteres e $\omega$-hidroxi-acilésteres do glicerol também foram identificados.

\section{CONCLUSÕES}

Embora a família Bromeliaceae conte com grande representatividade de espécies no Brasil, poucas foram estudadas química e farmacologicamente até o momento.

Algumas espécies de bromeliáceas são usadas na medicina tradicional contra problemas das vias respiratórias, como agentes antihelmínticos, antidiarréicos, antidiabéticos. O abacaxi, por exemplo, apresenta reconhecidas propriedades medicinais, agindo principalmente como estomáquico, depurativo, carminativo, diurético, tônico e antiinflamatório. ${ }^{28}$ Estudos farmacológicos com extratos das folhas de $A$. comosus mostraram efeitos antidiabético, anti-hiperlipidêmico e antioxidante. ${ }^{24,26}$ A espécie Tillandsia usneoides também apresentou significante efeito hipoglicêmico, onde o ácido 3-hidroxi-3-metilglutárico foi sugerido como agente hipoglicemiante no tratamento de diabetes mellitus. ${ }^{45}$ Outra espécie que tem apresentado potencial terapêutico é a Nidularium procerum, com destaque particular às atividades antialérgica, anti-inflamatória e potente atividade analgésica. ${ }^{42-44}$

Do ponto de vista químico, as espécies mais estudadas foram o Ananas comosus e a Tillandsia usneoides, sendo o primeiro certamente por questão de sua importância econômica mundial e o segundo em função de seu uso popular e ampla distribuição no continente americano. Nos poucos estudos pode-se constatar que duas classes de substâncias são características na família: flavonoides, especialmente com padrão de oxigenação na posição 6 , e cicloartanos.

A frequência e variedade de flavonas e flavonóis com oxigenação extra (hidroxilação, metoxilação ou glicosilação) na posição C-6 chamam a atenção, sendo que a predominância destes compostos nesta família é única dentro das monocotiledônias, ${ }^{72}$ podendo inclusive ser 
Tabela 2. Distribuição de flavonoides na família Bromeliaceae

\begin{tabular}{l} 
Espécies \\
\hline Subfamília Bromelioideae \\
Acanthostachys strobilacea \\
Aechemea bracteata \\
Aechemea bromeliifolia \\
Aechemea glomerata
\end{tabular}

Aechemea recurvata
Aechemea recurvata var. recurvata
Aechemea tillandsioides
Aechemea tillandsioides var. kienastii
Aechemea weilbachii var. leodiensis
Ananas comosus

Billbergia buchholtzii

Billbergia cv. fantasia

Billbergia vittata

Billbergia zebrina
Bromelia pinguin
Canistrum cyathiformi
Canistrum lindenii var. lindenii
Fascicularia kirchoffiana
Gravisia aquilega
Hohenbergia penduliflora

Neoregalia carolinae var. carolinae

Neoregalia cruenta

Neoregalia eleutheropetala

Neoregalia princeps

Neoregalia spectabilis

Nidularium fugens

Nidularium innocentii

compostos Ref.

35b 72

39o; 39q 73

35b 72

33a; 33b; 33c; 75

33d; 33e; 33h;

33j; 34; 35b;

39a; 39b; 39c;

39d

$\begin{array}{cc}\text { 39o; 39q; } & 73 \\ \text { 39s; 39t } & \\ \text { 39t } & 73\end{array}$

$\begin{array}{cc}\text { 39o; 39q } & 73 \\ 39 q & 73\end{array}$

39g; 39h; 39q 73

31a; 31b 76

33a; 33b; 77

$35 \mathrm{a} ; 35 \mathrm{c}$

33c; 33r; 33y 78

33k; 33r; 79

$33 \mathrm{~s} ; 33 \mathrm{y}$

35a; 35b; 35c; 80

36; 37

$35 \mathrm{c} \quad 81$

39o; 39q; 39s 73

39m; 39n; 39o; 73

39q; 39r; 39t

39o; 39q 73

33a; 33b; 33c; 75

33d; 33f; 33h;

33L; 35b; 39b;

39c; 39d

33b; 35b 72

33t; 35s; 35u 38

39o; 39q 73

35b; 35g 72

39q 73

35b 72

35b 72

39; 39o; 73

$39 \mathrm{~s} ; 39 \mathrm{t}$

39f; 39o; 39s 73

39o; 39q; 39s 73

39o; 39s; 39t 73

33b 72

35b 72

39o; 39q; 73

$39 \mathrm{~s} ; 39 \mathrm{t}$

$\frac{\text { Espécies }}{\text { Nidularium innocentii var. lineatum }}$

Nidularium procerum var. procerum

Nidularium aff. rutilans

Nidularium terminale

Portea petropolitana

Quesnelia liboniana

Streptocalyx longifolius

Streptocalyx poppigii

Subfamília Pitcairnioideae

Brocchinia reducta

Dykia nierdeleinii

Pitcairnia atrorubens

Pitcairnia corallina

Pitcairnia darblayana

Pitcairnia imbricata

Pitcairnia integrifolia

Pitcairnia poortimanii

Pitcairnia punicea

Pitcairnia rubriflora

Pitcairnia sprucei

Pitcairnia tabuliformis

Pitcairnia xanthocalyx

Puya alpestris

Puya chilensis

Puya laxa

Puya sp.

Puyopsis assurgens

Puyopsis coerulea

Puyopsis densiflora $\begin{array}{cc}\text { compostos } & \text { Ref. } \\ \text { 39g; 39L; 39o; } & 73\end{array}$ 39h; 39q

35b 72

35b 72

39o; 39q; 73

$39 \mathrm{~s} ; 39 \mathrm{t}$

35b; 35c; 35f; 72

$35 \mathrm{~h} ; 35 \mathrm{i}$

39o; 39s; 39t 73

35b 72

35b 72

39m; 39q 73

35b 72

39f 73

35b 72

33a; 33f; 33L; 72

33n; 330

33a; 33L; 33n 72

33n 72

33m; 33o; 35b 72

39e 73

33L; 33m; 33n; 72

33o; 33q

331; 35b 72

33a; 33d; 33h; 72

33n; 33o; 35a;

$35 \mathrm{~b} ; 35 \mathrm{e}$;

35f; $35 \mathrm{~g}$

33h; 33n; 33o; 72

35a; 35b; 35e;

35f; $35 \mathrm{~g}$

39e 73

39q; 39s; 39t 73

33b; 33j; 72

$33 n ; 330$

39j; 39k; 39p 74

39i; 39j; 39p 74

39f; 39n 73

33n; 33o; 33 v; 72

$33 x ; 35 g$

39f; 39k; 39o 74

39f; 39j; 39k; 74

$390 ; 39 p$

39f; 39j; 39k; 74

$390 ; 39$ p

39k; 39o

3

2

72

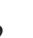

2

3

2

72

2

Nidularium innocentii var. innocentii $\quad$ 35b; 35c; 35d 72 
Tabela 2. continuação

\begin{tabular}{|c|c|c|c|c|c|}
\hline Espécies & compostos & Ref. & Espécies & compostos & Ref. \\
\hline Puyopsis ferruginea & 39f; 39j; 39o & 74 & Tillandsia flabelatta & $39 \mathrm{~m} ; 39 \mathrm{~s} ; 39 \mathrm{t}$ & 73 \\
\hline Puyopsis floccosa & 39i; 39k; 39p & 74 & Tillandsia lindeniana & 33b; 33n; 35b & 72 \\
\hline Puyopsis laxa & $\begin{array}{l}\text { 39f; 39j; 39k; } \\
\text { 39o; 39p }\end{array}$ & 74 & Tillandsia monadelpha & $390 ; 39 t$ & 73 \\
\hline \multirow{2}{*}{ Puyopsis spathacea } & \multirow{2}{*}{$\begin{array}{c}\text { 39f; 39j; 39k; } \\
39 \mathrm{o} ; 39 \mathrm{p}\end{array}$} & \multirow{2}{*}{74} & Tillandsia morreniana & 33b; 33n & 72 \\
\hline & & & Tillandsia purpurea & $35 \mathrm{k} ; 35 \mathrm{t} ; 35 \mathrm{v}$ & 82 \\
\hline Puyopsis venusta & 39j; 39k; 39p & 74 & Tillandsia recurvata & $32 \mathrm{c}$ & 49 \\
\hline \multicolumn{3}{|l|}{ Subfamília Tillandsioideae } & Tillandsia streptocarpa & $35 \mathbf{b}$ & 53 \\
\hline Catopsis berteroniana & $33 b ; 38$ & 72 & Tillandsia tricolor & $39 o ; 39 s ; 39 t$ & 73 \\
\hline Criptanthus bahianus & 39o; 39s; 39t & 73 & \multirow[t]{4}{*}{ Tillandsia usneoides } & \multirow{2}{*}{$\begin{array}{c}\text { 35b; 35g; 35m; } \\
\text { 35p; 35q; 35r } \\
\text { 35j }\end{array}$} & \multirow[t]{2}{*}{72} \\
\hline Criptanthus bivittatus & 39q; 39s; 39t & 73 & & & \\
\hline Criptanthus $x$. osyanthus & 39q; 39s; 39t & 73 & & \multirow{2}{*}{$\begin{array}{l}35 q \\
35 r\end{array}$} & $\begin{array}{l}83 \\
84\end{array}$ \\
\hline Criptanthus sinuosus & 39o; 39q; 39s & 73 & & & 45 \\
\hline Criptanthus zonatus forma zonatus & $39 q$ & 73 & Tillandsia utriculata & 33r; 33w; 33u & 85 \\
\hline Greigia sphacelata & $32 \mathrm{a} ; 32 \mathrm{~b}$ & 58 & Vriesia imperialis & $35 b ; 35 L$ & 72 \\
\hline Guzmania zahnii & 39m; 39s & 73 & Vriesia inflata & 39s; 39t & 73 \\
\hline Tillandsia aeranthos & 35b & 72 & Vriesia platynema & 39f; 39i & 73 \\
\hline Tillandsia bulbosa & $35 \mathrm{~L} ; 35 \mathrm{n}$ & 72 & Vriesia regina & 35b; 35f; 35L; & 72 \\
\hline Tillandsia cyanea & $33 n$ & 72 & & 35n; 350 & \\
\hline Tillandsia fasciculata & $35 \mathrm{~b} ; 35 \mathrm{~m} ; 35 \mathrm{p}$ & 72 & Vriesia sanguinolenta & $33 p$ & 86 \\
\hline
\end{tabular}

Tabela 3. Ocorrência de flavonoides com e sem oxigenação na posição C-6 nas três subfamílias de Bromeliaceae

\begin{tabular}{|c|c|c|c|c|c|c|}
\hline \multirow[t]{2}{*}{ Flavonoides } & \multicolumn{2}{|c|}{ Bromelioideae } & \multicolumn{2}{|c|}{ Pitcairnioideae } & \multicolumn{2}{|c|}{ Tillandsioideae } \\
\hline & $\mathrm{C}_{6}-\mathrm{H}$ & $\mathrm{C}_{6}-\mathrm{OR}$ & $\mathrm{C}_{6}-\mathrm{H}$ & $\mathrm{C}_{6}-\mathrm{OR}$ & $\mathrm{C}_{6}-\mathrm{H}$ & $\mathrm{C}_{6}-\mathrm{OR}$ \\
\hline Flavanona & -- & -- & -- & -- & -- & 3 \\
\hline Flavona & 5 & 4 & 9 & 23 & 3 & 7 \\
\hline Flavonol & 23 & 2 & 14 & -- & 10 & 14 \\
\hline
\end{tabular}<smiles>[Y]c1ccc(/C=C/C(N)=O)cc1[2H]</smiles><smiles>O=C(/C=C/c1ccc(O)cc1)OC1CC(C(=O)O)C(O)[C@H](O)[C@H]1O</smiles>

40a: $\mathrm{R}=\mathrm{OH} ; \mathrm{R}^{1}=\mathrm{R}^{2}=\mathrm{H}$

$\underline{40 \mathbf{b}}: \mathrm{R}=\mathrm{R}^{1}=\mathrm{OH} ; \mathrm{R}^{2}=\mathrm{H}$

40ㄷ: $\mathrm{R}=\mathrm{R}^{1}=\mathrm{R}^{2}=\mathrm{OH}$

40. $\mathrm{R}=\mathrm{R}^{1}=\mathrm{OH} ; \mathrm{R}^{2}=\mathrm{OCH}_{3}$

40ㄹ $: \mathrm{R}=\mathrm{R}^{2}=\mathrm{OH} ; \mathrm{R}^{1}=\mathrm{OCH}_{3}$

40f: $\mathrm{R}=\mathrm{CH}_{2} \mathrm{CH}_{3} ; \mathrm{R}^{1}=\mathrm{R}^{2}=\mathrm{OH}$

40 $\mathrm{g}: \mathrm{R}=\left(\mathrm{CH}_{2}\right)_{21} \mathrm{COOH} ; \mathrm{R}^{1}=\mathrm{OH} ; \mathrm{R}^{2}=\mathrm{OCH}_{3}$<smiles>O=C(/C=C/c1ccc(O)cc1)OC1C(O)CC(O)(OC(=O)/C=C/c2ccc(O)cc2)CC1O</smiles><smiles>O=C(/C=C/c1ccc(O)cc1)OC1CC(O)(C(=O)O)C[C@H](O)[C@H]1OC(=O)/C=C/c1ccc(O)c(O)c1</smiles>

Figura 7. Ácidos cinâmicos e derivados relatados na família Bromeliaceae utilizada como indicador de avanço filogenético para distinguir as três subfamílias de Bromeliaceae. Neste sentido, quimiotaxonomicamente a subfamília Bromelioideae apareceria como a mais primitiva, seguida da subfamília Pitcairnioideae e a mais evoluída seria a subfamília Tillandsioideae. Taxonômica e anatomicamente, a subfamília Tillandsioideae é vista como a mais evoluída dentro da família Bromeliaceae. Entretanto, ainda há muitas incertezas quanto à mais primitiva. ${ }^{72}$ Antocianinas triglicosiladas (cianidinas 3,5,3' 'triglicosiladas e delfinidinas 3,7,3'-triglicosiladas) identificadas nesta família também apresentam grau de complexidade estrutural a ser considerado dentro dos flavonoides.

Apesar do número de espécies avaliadas que apresentam triterpenos em sua composição ser pequeno, a variedade de cicloartanos e sua predominância na subfamília Tillandsioideae também chamam a atenção. Embora nenhuma correlação com outras famílias tenha sido proposta ainda, a ocorrência desta classe dentro das subfamílias eventualmente poderia servir como indicativo taxonômico.

De modo geral, a diversidade de metabólitos e as atividades biológicas observadas em Bromeliaceae justificariam o interesse no estudo de espécies desta família. Compostos como os diglicerídeos cinâmicos (51c-h), diterpenoides filocladanos $(\mathbf{2 6}, \mathbf{2 7})$, derivados 


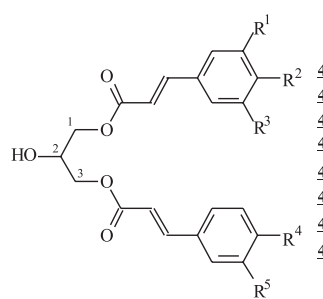

45a: $\mathrm{R}^{1}=\mathrm{R}^{2}=\mathrm{R}^{3}=\mathrm{R}^{4}=\mathrm{R}^{5}=\mathrm{H}$ 45 : $: R^{1}=R^{3}=R^{5}=H ; R^{2}=R^{4}=O H ;$ $45 \mathrm{c}: \mathrm{R}^{3}=\mathrm{R}^{5}=\mathrm{H} ; \mathrm{R}^{1}=\mathrm{R}^{2}=\mathrm{R}^{4}=\mathrm{OH}$ 45d: $R^{1}=\mathrm{OCH}_{3} ; \mathrm{R}^{3}=\mathrm{R}^{5}=\mathrm{H} ; \mathrm{R}^{2}=\mathrm{R}^{4}=\mathrm{OH}$; $45 \mathrm{e}: \mathrm{R}^{3}=\mathrm{H} ; \mathrm{R}^{1}=\mathrm{R}^{2}=\mathrm{R}^{4}=\mathrm{R}^{5}=\mathrm{OH} ;$ 45f: $\mathrm{R}^{1}=\mathrm{OCH}_{3} ; \mathrm{R}^{2}=\mathrm{R}^{4}=\mathrm{R}^{5}=\mathrm{OH} ; \mathrm{R}^{3}=\mathrm{H}$ 45 : $\mathrm{R}^{1}=\mathrm{R}^{2}=\mathrm{R}^{3}=\mathrm{R}^{4}=\mathrm{OH} ; \mathrm{R}^{5}=\mathrm{OCH}_{3}$<smiles>Cc1cc(/C=C/C(=O)OCC(O)CO)ccc1O</smiles>

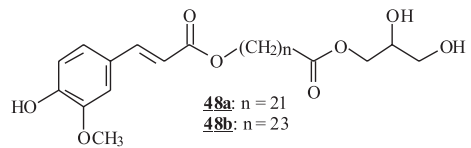

Figura 8. Mono- e diglicerídeos fenilpropenatos relatados na família Bromeliaceae<smiles>[Y2]c1cc(C2OCC3C(c4cc([Z])c(O)c(OC)c4)OCC23)cc(OC)c1O</smiles><smiles>COc1cc(CC(CO)C(Cc2ccc(O)c(OC)c2)Cc2ccc(CC3COC(=O)C3Cc3ccc(O)c(OC)c3)c(OC)c2)ccc1O</smiles>

Figura 9. Lignanas identificadas na família Bromeliaceae<smiles>O=C(O)c1cc(O)c(O)c(O)c1</smiles><smiles>O=C(Oc1cc(C(=O)OC2C3OC(O)C(C(OC(=O)c4cc(O)c(O)c(O)c4)O3)C2C(O)O)cc(O)c1O)c1cc(O)c(O)c(O)c1</smiles>

Figura 10. Outros compostos fenólicos relatados na família Bromeliaceae

S-derivados (62-64) do álcool sinapílico, alguns flavonoides, cuja ocorrência na natureza ainda é bastante restrita, evidenciam que muitas substâncias com estruturas diferenciadas poderiam surgir se mais espécies desta família fossem estudadas.

Enfim, acreditamos que esta fascinante família apresente boas perspectivas de estudo, com interessante potencial químico e farmacológico a ser descoberto.

\section{AGRADECIMENTOS}

À Universidade Paranaense (UNIPAR) pelo apoio financeiro recebido e à Diretoria Executiva de Gestão da Pesquisa e da PósGraduação (DEGPP) pelo incentivo aos programas de pesquisa.<smiles>[R]c1ccc2[nH]c3c(c2c1)CC([R])NC3[R]</smiles><smiles></smiles>

58a: $\mathrm{R}^{1}=\mathrm{CH}_{3} ; \mathrm{R}^{2}=\mathrm{H} ; \mathrm{R}^{3}=\mathrm{OH}$ 58b: $R^{1}=H ; R^{2}=\mathrm{COOH} ; R^{3}=\mathrm{H}$ 58c: $\mathrm{R}^{1}=\mathrm{CH}_{3} ; \mathrm{R}^{2}=\mathrm{COOH} ; \mathrm{R}^{3}=\mathrm{H}$ 58d: $\mathrm{R}^{1}=\mathrm{CH}_{3} ; \mathrm{R}^{2}=\mathrm{R}^{3}=\mathrm{H}$

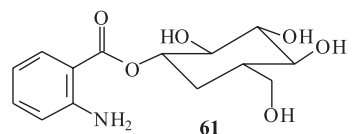<smiles>[R]NNC(CSCC=Cc1cc(OC)c(O)c(OC)c1)C([R])=O</smiles>

62: $S$-sinapil-L-cisteína $\mathrm{R}^{\mathrm{l}}=\mathrm{OH} ; \mathrm{R}^{2}=\mathrm{H}$

63: $S$-sinapil-glutationa $\mathrm{R}^{1}=-\mathrm{NHCH}_{2} \mathrm{COOH}$

$\mathrm{R}^{2}=-\mathrm{COCH}_{2} \mathrm{CH}_{2} \mathrm{CH}\left(\mathrm{NH}_{2}\right) \mathrm{COOH}$

64: $N$-L- $\gamma$-glutamil- $S$-sinapil-L-cisteína $\mathrm{R}^{1}=-\mathrm{NHCH}_{2} \mathrm{COOH}$ $\mathrm{R}^{2}=\mathrm{H}$

Figura 11. Compostos nitrogenados identificados na família Bromeliaceae

\section{REFERÊNCIAS}

1. Benzing, D. H.; Bromeliaceae: Profile of an adaptive radiation, ed.; Cambridge University Press: New York, 2000.

2. Benzing, D. H.; The Biology of Bromeliads, Mad River Press: Eureka, California, 1980.

3. Madisom, M.; Sellbiana 1977, $2,1$.

4. Benzing, D. H.; Henderson, K.; Kessel, B.; Sulak, J.; Amer. J. Bot. 1976, 63, 1009 .

5. http://www.bsi.org, acessada em Dezembro 2007.

6. Pita, P.; Menezes, N. L.; Rev. Bras. Bot. 2002, 1, 25.

7. Bennett, B.C. Em Ethnobotany of Bromeliaceae in Bromeliaceae: Profile of an adaptative radiation; Benzing, D. H., ed.; Cambridge University: Cambridge, 2000, cap 14.

8. Corrêa, M. P.; Dicionário das Plantas Úteis do Brasil e das Exóticas Cultivadas, IBDF: Rio de Janeiro, 1984, vol. 2, p.16.

9. Souza, V. C.; Lorenzo, H.; Botânica Sistemática: guia ilustrado para identificação das famílias de Angiospermas da flora brasileira, Ed. Plantarum: São Paulo, 2005, p.162-166.

10. Cathcart, D. J.; Florida Entomologist 1995, 78, 16.

11. Maurer, H. R.; Cell. Mol. Life Sci. 2001, 58, 1234; Tysnes, B. B.; Maurer, H. R.; Porwol, T.; Probst, B.; Bjerkvig, R.; Hoover, F.; Neoplasia 2001, 3, 469; Metzig, C.; Grabowska E.; Eckert, K.; Maurer, H. R.; In Vivo 1999, 13, 7.

12. Freiman, L. O.; Sabaa Srur, A. U.; Cienc. Tecn. Alim. 1999, 19, 170.

13. Vallés, D.; Furtado, S.; Cantera, A. M. B.; Enz. Microb. Technol. 2007, 40, 409; Payrol, J. A.; Obregón, W. D.; Natalucci, C. L.; Caffini, N. O.; Fitoterapia 2005, 76, 540; Taira, T.; Toma, N.; Ishihara, M.; Biosci. Biotechnol. Biochem. 2005, 69, 189; López, L. M. I.; Sequeiros, C.; Natalucci, C. L.; Brullo, A.; Maras, B.; Barra, D.; Caffini, N. O.; Prot. Exp. Purific. 2000, 18, 133; Pardo, M. F.; López, L. M. I.; Canals, F.; Avilés, F. X.; Natalucci, C. L.; Caffini, N. O.; J. Agric. Food Chem. 2000, 48, 3795.

14. Bezerra, F.C.; Paiva, W. O.; Agronegócios de Flores no Estado do Ceará: Situação Atual e Perspectivas, Embrapa Agroindústria Tropical, 1997.

15. Malm, O.; Fonseca, M. D.; Miguel, P. H.; Bastos, W. R.; Pinto, F. N.; Sci. Total Environ. 1998, 213, 57

16. Figueiredo, A. M. G.; Alcala, A. L.; Ticianelli, R. B.; Domingos, M.; Saiki, M.; J. Radioanal. Nucl. Chem. 2004, 259, 59; Amado, G. M.; Andrade, L. R.; Farina, M.; Malm, O.; Atmos. Environ. 2002, 36, 881; Brighigna, L.; Papini, A.; Mosti, S.; Cornia, A.; Bocchini, P.; Galletti, G.; Rev. Biol. Trop. 2002, 50, 577; Figueiredo, A. M. G.; Saiki, M.; Ticianelli, R. B.; Domingos, M.; Alves, E. S.; Markert, B.; 
J. Radioanal. Nucl. Chem. 2001, 249, 391; Calasans, C. F.; Malm, O.; Bromelia 1994, 1, 7.

17. Schrimpff, E.; Water, Air, Soil Pollut. 1984, 21, 279.

18. Flores, F. E. V.; Tübinger Geor. Studien. 1987, 96, 79.

19. Brighigna, L.; Ravanelli, M.; Minelli, A.; Ercoli, L. B.; Sci. Total Environ. 1997, 198, 175.

20. Pignata, M. L.; Gudino, G. L.; Wannaz, E. D.; Pla, R. R.; Gonzalez, C. M.; Carreras, H. A.; Orellana, L.; Environ. Pollut. 2002, 120, 59.

21. Zah, R.; Hischier, R.; Leão, A. L.; J. Cleaner Prod. 2007, 15, 1032.

22. Marques, G.; Gutiérrez, A.; Del Rio, J. C.; J. Agric. Food Chem. 2007, $55,1327$.

23. Lorenzi, H.; Matos, F. J. A.; Plantas medicinais no Brasil: nativas e exóticas cultivadas, Ed. Plantarum: São Paulo, 2002.

24. Xie, W.; Wang, W.; Su, H.; Xing, D.; Pan, Y.; Du, L.; Comp. Biochem. Physiol. 2006, 143, 429.

25. Pakrashi, S. C.; Achari, B.; Majumdar, P. C.; Indian J. Chem. 1975, 13, 755.

26. Xie, W. D.; Xing, D. M.; Sun, H.; Wang, W.; Ding, Y.; Du, L. J.; Am. J. Chin. Med. 2005, 33, 95.

27. Xie, W.; Wang, W.; Su, H.; Xing, D.; Cai, G.; Du, L.; J. Pharmacol. Sci. 2007, 103, 267.

28. Duke, J. A.; Bogenschutz-Godwin, M. J.; duCellier, J.; Duke, P.-A. K.; Handbook of medicinal herbs, $2^{\text {nd }}$ ed., CRC Press: Boca Raton, 2000, p. 570.

29. Cruz, G. L.; Dicionário de Plantas Úteis do Brasil, $5^{\text {a }}$ ed., Ed. Bertrand: Rio de Janeiro, 1995.

30. Rodrigues, V. E. G.; Carvalho, D. A.; Rev. Bras. Pl. Med. 2007, 2, 17; Rodrigues, V. E. G.; Carvalho, D. A.; Ciênc. Agrotec. 2001, 1, 102.

31. Jorge, L. I. F.; Ferro, V. O.; Rev. Farm. Bioquím. USP 1993, 29, 69.

32. Andrighetti-Frohner, C. R.; Sincero, T. C. M.; Silva, A. C.; Savi, L. A.; Gaido, C. M.; Bettega, J. M. R.; Mancini, M.; Almeida, M. T. R.; Barbosa, R. A.; Farias, M. R.; Barardi, C. R. M.; Simões, C. M. O.; Fitoterapia 2005, 75, 374.

33. Garlet, T. M. B.; Irgang, B. E.; Rev. Bras. Pl. Med. 2001, 4, 9.

34. Dorigoni, P. A.; Ghedini, P. C.; Fróes, L. F.; Ethur, A. B. M.; Baldisserotto, B.; Burger, M. E.; Almeida, C. E.; Lopes, A. M. V.; Záchia, R. A.; Rev. Bras. Pl. Med. 2001, 4, 69.

35. Reitz, R.; Plantas Medicinais de Santa Catarina. Anais botânicos do herbário Barbosa Rodrigues. Itajaí, 1950, 2, 71.

36. Agra, M. F.; Freitas, P. F.; Barbosa-Filho, J. M.; Rev. Bras. Pharmacog. 2007, 17, 114

37. Franco, E. A. P.; Barros, R. F. M.; Rev. Bras. Pl. Med. 2006, 8, 78.

38. Raffauf, R. F.; Menachery, M. D.; Le Quesne, P. W.; Arnold, E. V.; Clardy, J.; J. Org. Chem. 1981, 46, 1094.

39. Camacho-Hernández, I. L.; Cháez-Velásquez, J. A.; Uribe-Beltrán, M. J.; Rios-Morgan, A.; Delgado-Vargas, F.; Fitoterapia 2002, 73, 411.

40. Payrol, J. A.; Martinez, M. M.; Rev. Cubana Farm. 2000, 34, 181.

41. Geran, R. I.; Greenberg, N. H.; MacDonald, M. M.; Schumacher, A. M.; Abbott, B. J.; Cancer Chemother. Rep. 1972, 3, 1.

42. Vieira-de-Abreu, A.; Amendoeira, F. C.; Gomes, G. C.; Zanon, C.; Cheider, L. C.; Figueiredo, M. R.; Kaplan, M. A. C.; Frutuoso, V. S.; Castro-Faria-Neto, H. C.; Weller, P. F.; Bandeira-Melo, C.; Bozza, P. T.; Int. Immunopharm. 2005, 5, 1966.

43. Amendoeira, F. C.; Frutuoso, V. S.; Zanon, C.; Cheider, L. C.; Figueiredo, M. R.; Kaplan, M. A. C.; Bandeira-Melo, C.; Bozza, P. T.; Castro-Faria-Neto, H. C.; Phytomedicine 2005, 12, 78.

44. Amendoeira, F. C.; Frutuoso, V. S.; Zanon, C.; Cheider, L. C.; Figueiredo, M. R.; Kaplan, M. A. C.; Bandeira-Melo, C.; Bozza, P. T.; Castro-Faria-Neto, H. C.; Biol. Pharm. Bull. 2005, 28, 1010.

45. Whiterup, K. M.; McLaughlin, J. L.; Judd, R. L.; Ziegler, M. H.; Medon, P. J.; Keller, W. J.; J. Nat. Prod. 1995, 58, 1285.

46. Feurt, S.; Fox, L. E.; J. Am. Pharm. Assoc. Sci. 1952, 41, 453.

47. Weld, J. T.; Proc. Soc. Exp. Biol. Med. 1945, 59, 40.
48. Rios, A. R.; Khan, B.; J. Bromeliad Soc. 1998, 75.

49. Queiroga, M. A.; Andrade, L. M.; Florêncio, K. C.; Agra, M. F.; Silva, M. S.; Barbosa-Filho, J. M.; Cunha, E. V. L.; Fitoterapia 2004, 75, 423.

50. Alonso Paz, E.; Cerdeiras, M. P.; Fernandez, J.; Ferreira, F.; Moyna, P.; Soubes, M.; Vazquez, A.; Vero, S.; Zunino, L.; J. Ethnopharmacol. 1995, $45,67$.

51. Filipov, A.; J. Ethnopharmacol. 1994, 44, 182.

52. Weniger, B.; Rouzier, M.; Dauguilb, R.; Henrys, D.; Henrys, R.; Anton, R.; J. Ethnopharmacol. 1986, 17, 13.

53. Delaporte, R. H.; Sarragiotto, M. H.; Takemura, O. S.; Sanchez, G. M.; Filho, P. D.; Nakamura, C. V.; J. Ethnopharmacol. 2004, 95, 229.

54. Elss, S.; Preston, C.; Hertzig, C.; Heckel, F.; Richling, E.; Schreier, P.; LWT 2005, 38, 263; Tokitomo, Y.; Steinhaus, M.; Buttner, A.; Schieberle, P.; Biosci. Biotechnol. Biochem. 2005, 69, 1323; Preston, C.; Richling, E.; Elss, S.; Appel, M.; Heckel, F.; Hartlieb, A.; Schreier, P.; J. Agric. Food Chem. 2003, 51, 8027; Umano, K.; Hagi, Y.; Nakahara, K.; Shoji, A.; Shibamoto, T.; J. Agric. Food Chem. 1992, 40, 599; Wu, P.; Kuo, M.-C.; Hartman, T. G.; Rosen, R. T.; Ho, C.-T.; J. Agric. Food Chem. 1991, 39, 170; Haagen-Smit, A. J.; Kirchner, J. G., Prater, A. N.; Deasy, C. L.; J. Am. Chem. Soc. 1945, 67, 1646; Haagen-Smit, A. J.; Deasy, C. L.; Prater, A. N.; J. Am. Chem. Soc. 1945, 67, 1651.

55. Wang, C.; Du, L.; Ding, Y.; Xing, D.; Wang, R.; Ma, C.; Wang, X.; Chen, Y.; Faming Zhuanli Shenqing Gonkai Shuomingshu 2005, (CA 144: 3457).

56. Wang, W.; Ding, Y.; Xing, D. M.; Wang, J. P.; Du, L. J.; China J. Chinese Mat. Med. 2006, 31, 1242 (CA 148: 410932).

57. Takata, R. H.; Scheuer, P. J.; Tetrahedron 1976, 32, 1077.

58. Flagg, M. L.; Wachter, G. A.; Davis, A. L.; Montenegro, G.; Timmermann, B. N.; J. Nat. Prod. 2000, 63, 1689

59. Marker, R.; Wagner, R. B.; Ulshafer, P. R.; Wittbecker, E. M.; Goldsmith, D. P. J.; Ruof, C. H.; J. Am. Chem. Soc. 1943, 1199.

60. Cantillo-Ciau, Z.; Mena-Rejon, G. J.; Quintero-Marmol, E.; JimenezDiaz, A.; Quijano L.; Z. Naturforsch C 2003, 58, 649.

61. Cantillo-Ciau, Z.; Brito-Loeza, W.; Quijano, L.; J. Nat. Prod. 2001, 64, 953.

62. Caiado, J.; Lucietto, C. L.; Delaporte, R. H.; Laverde Jr.; A.; Chem. Nat. Compd., submetido.

63. Cabrera, G. M.; Seldes, A. M.; J. Nat. Prod. 1995, 58, 1920.

64. Cabrera, G. M.; Gallo, M.; Seldes, A. M.; J. Nat. Prod. 1996, 59, 343.

65. Djerassi, C.; McCrindle, R.; J. Chem. Soc. 1962, 10, 4034.

66. McCrindle, R.; Djerassi, C.; Chem. Ind. 1961, 19, 1311.

67. Cabrera, G. M.; Seldes, A. M.; Phytochemistry 1997, 45, 1019.

68. Atallah, A. M.; Nicholas, H. J.; Phytochemistry 1971, 10, 3139.

69. Cabrera, G. M.; Gallo, M.; Seldes, A. M.; Phytochemistry 1995, 39, 665.

70. Herz, W.; Watanabe, K.; Kulanthaivel, P.; Blount, J. F.; Phytochemistry 1985, 24, 2645

71. Marker, R.; Wagner, R. B.; Ulshafer, P. R.; Wittbecker, E. M.; Goldsmith, D. P. J.; Ruof, C. H.; J. Am. Chem. Soc. 1947, 2167.

72. Williams, C. A.; Phytochemistry 1978, 17, 729.

73. Sato, N.; Harborne, J. B.; Phytochemistry 1983, 22, 1735.

74. Scogin, R.; Biochem. Syst. Ecol. 1985, 13, 387.

75. Ashtakala, S. S.; J. Am. Soc. Hort. Sc. 1975, 100, 546.

76. Yi, X.; Wei, B.; Tenj, J.; Gao, C.; Shipin. Yu Fajiao Gongye 2006, 32, 99 (CA 147: 405297).

77. Ma, C.; Xiao, S.; Li, Z.; Wang, W.; Du, L.; J. Chromatogr., A 2007, $1165,39$.

78. Xing, D.; Wang, W.; Wang, R.; Zhao, Y.; Chen, Y.; Du, L; Ding, Y.; Faming Zhuanli Shenqing Gonkai Shuomingshu 2005 (CA 147: 74743).

79. Franke, A. A.; Custer, L. J.; Arakaki, C.; Murphy, S. P.; J. Food Comp. Anal. 2004, 17, 1.

80. Lako, J.; Trenerry, V. C.; Wahlqvist, M.; Wattanapenpaiboon, N.; Sotheeswaran, S.; Premier, R.; Food Chem. 2007, 101, 1727. 
81. Larrauri, J. A.; Rupérez, P.; Saura-Calixto, F.; J. Agric. Food Chem. 1997, 45, 4028.

82. Arslanian, R. L.; Stermitz, F. R.; Castelo, L.; J. Nat. Prod. 1986, 49, 1177.

83. Wollenweber, E.; Mann, K.; Roitmann, J. N.; Z. Naturforsch C 1992, 47, 638 .

84. Lewis, D. S.; Mabry, T. J.; Phytochemistry 1977, 16, 1114.

85. Ulubelen, A.; Mabry, T. J.; Rev. Latinoamer. Quim. 1982, 13, 35.

86. Bringmann, G.; Ochse, M.; Zotz, G.; Peters, K.; Peters, E. M.; Brun, R.; Schauler, J.; Phytochemistry 2000, 53, 965.

87. Takata, R. H.; Scheuer, P. J.; Lloydia 1976, 39, 409.

88. Sutherland, G. K.; Gortner, W. A.; Australian J. Chem. 1959, 12, 240.

89. Koshino, H.; Terada, S. I.; Yoshihara, T.; Kakamura, S.; Shimanuki, T.; Sato, T.; Tajimi, A.; Phytochemistry 1988, 27, 1333; Cooper, R.; Gottlieb, H. E.; Lavie, D.; Phytochemistry 1978, 17, 1673.

90. Jang, D. S.; Cuendet, M.; Fong, H. H. S.; Pezzuto, J. M.; Kinghorn, A. D.; J. Agric. Food Chem. 2004, 52, 2218; Mimaki, Y.; Sashida, Y.; Simomura, H.; Phytochemistry 1989, 28, 3453; Shimomura, H.; Sashida, Y.; Mimaki, Y.; Chem. Pharm. Bull. 1988, 36, 4841; Shimomura, H.; Sashida, Y.; Mimaki, Y.; Iitaka, Y.; Chem. Pharm. Bull. 1988, 36, 2430; Shimomura, H.; Sashida, Y.; Mimaki, Y.; Phytochemistry 1987, 26, 844.
91. Shirota, O.; Sekita, S.; Satake, M.; Ni, Y.; Weiyi, H.; J. Nat. Prod. 1996, $59,242$.

92. Hiroyuki, K.; Teruhiko, Y.; Satoshi, T.; Tennen Yuki Kagobutsu Toronkai Koen Yoshishu 1989, 31, 244.

93. Du, L; Wang, W.; Xing, D.; Xiang, L.; Wang, X.; Faming Zhuanli Shenqing Gonkai Shuomingshu 2005, (CA 147: 402181).

94. Shen, J.; Xie, W.; Wang, W.; Ding, Y.; Du, L.; Zhongguo Zhongyao Zazhi 2007, 32, 813 .

95. Delaporte, R. H.; Guzen, K.P.; Laverde Jr., A.; Santos, A. R.; Sarragiotto, M. H.; Biochem. Syst. Ecol. 2006, 34, 599.

96. Parada, F.; Krajewski, D.; Hederich, M.; Duque, C.; Schreier, P.; Nat. Prod. Lett. 1995, 7, 69.

97. Herraiz, T.; Galisteo, J.; J. Agric. Food Chem. 2003, 51, 7156.

98. Peñalvo, J. L.; Haajanen, K. M.; Botting, N.; Adlercreutz, H.; J. Agric. Food Chem. 2005, 53, 9342.

99. Adrian-Romero, M.; Blunden, G.; Biochem. Syst. Ecol. 2001, 29, 305.

100. Parada, F.; Krajewski, D.; Duque, C.; Jarger, E.; Hederich, M.; Schreier, P.; Phytochemistry 1996, 42, 871.

101. Mullen, W.; Marks, S. C.; Crozier, A.; J. Agric. Food Chem. 2007, 55, 3148; Wen, L.; Wrolstad, R. E.; Hsu, V. L.; J. Agric. Food Chem. 1999, 47,850 . 